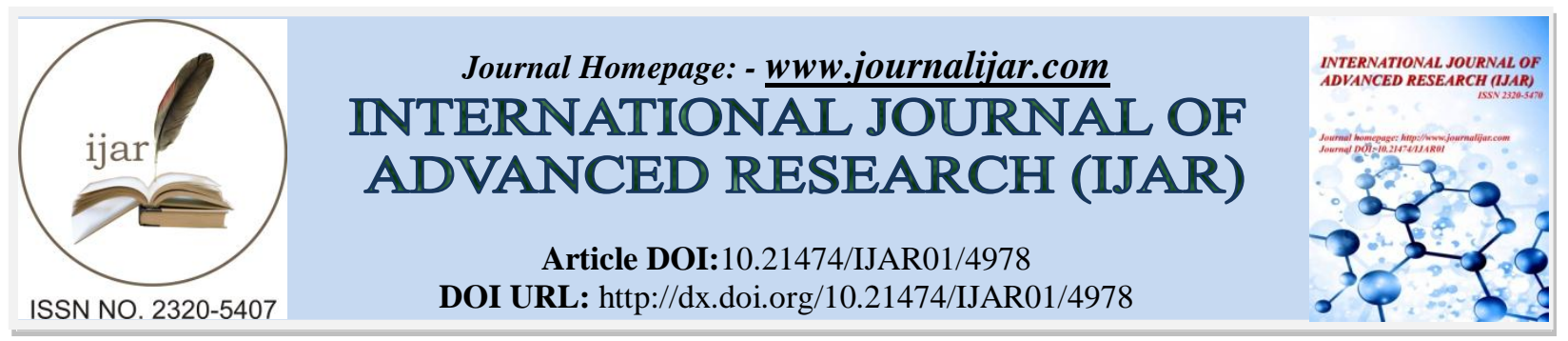

RESEARCH ARTICLE

\title{
ON NANO GENERALIZED $\beta$ LOCALLY CLOSED SETSAND NANO GENERALIZED $\beta$ LOCALLY CLOSED CONTINUOUS FUNCTIONS IN NANO TOPOLOGICAL SPACES.
}

S. B. Shalini, G. Sindhu and K. Indirani.

Department of Mathematics, Nirmala College for Women, Coimbatore, Tamil Nadu, India.

\section{Manuscript Info}

\section{Manuscript History}

Received: 27 May 2017

Final Accepted: 29 June 2017

Published: July 2017

Key words:-

$\mathrm{N} \beta$ LC set, NG $\beta$ LC set, $\mathrm{N} \beta$ LC

continuous, NG $\beta$ LC continuous and

NG $\beta$ LC irresolute functions.

\section{Abstract}

The aim of this paper is to introduce Nano generalized $\beta$ locally closed set and Nano generalized locally closed continuous function in Nano topological spaces and also we introduce and study the relation between Nano generalized $\beta$ locally closed irresolute function and Nano generalized $\beta$ locally closed continuous function.

Copy Right, IJAR, 2017,. All rights reserved.

\section{Introduction:-}

In general, continuous function is one of the core concepts in topology. Abd El Monsef et al. [1] introduced the notion of $\beta$-open sets and $\beta$-continuous mappings in topology.Balachandran [2] introduced generalized locally closed set and GLC continuous in topology. The concept of Nano topology was introduced by LellisThivagar [4] which was defined in terms of approximations and boundary regions of a subset of a universe using an equivalence relation on it and he also defined Nano continuous functions, Nano open maps, Nano closed maps and Nano homeomorphisms and their representations in terms ofNano interior and Nano closure.In this paper we introduce Nano generalized $\beta$ locally closed sets, Nano generalized $\beta$ locally closed continuous function and Nano generalized $\beta$ locally closed irresolute function and study some of its relation between them.

\section{Preliminaries:-}

Definition:- 2.1[4] Let $U$ be the universe, $R$ be an equivalence relation on $U$ and $\tau_{R}(X)=\left\{U, \phi, L_{R}(X), U_{R}(X), B_{R}(X)\right\}$ where $X \subseteq U$. Then $\tau_{R}(X)$ satisfies the following axioms:

i) $U$ and $\phi \in \tau_{R}(X)$.

ii) The union of the elements of any sub collection of $\tau_{R}(X)$ is in $\tau_{R}(X)$.

iii) The intersection of the elements of any finite sub collection of $\tau_{R}(X)$ is in $\tau_{R}(X)$.

Then $\tau_{R}(X)$ is a topology on $U$ called the Nano topology on $U$ with respect to $X,\left(U, \tau_{R}(X)\right)$ is called the Nano topological space. 
Definition:- 2.2 [4] If $\left(U, \tau_{R}(X)\right)$ is a Nano topological space with respect $X$ where $X \subseteq U$ and if $A \subseteq U$, then

1. The Nano interior of a set $A$ is defined as the union of all Nano open subsets contained in $A$ and is denoted by $N \operatorname{int}(A) . N \operatorname{int}(A)$ isthe largest Nano open subset of $A$.

2. The Nano closure of a set $A$ is defined as the intersection of all Nano closed sets containing $A$ and is denoted by $\operatorname{Ncl}(A) . \operatorname{Ncl}(A)$ is the smallest Nano closed set containing $A$.

Definition:- 2.3 [2] A function $f:(X, \tau) \rightarrow(Y, \sigma)$ is called continuous if $f^{-1}(V)$ is open in $(X, \tau)$ for every open set $V$ in $(Y, \sigma)$.

(ii) $\quad \beta$ (Semi pre) continuous if $f^{-1}(V)$ is $\beta$ (semi pre) open in $(X, \tau)$ for every open set $V$ in $(Y, \sigma)$.

(iii) gcontinuous if $f^{-1}(V)$ is g open in $(X, \tau)$ for every open set $V$ in $(Y, \sigma)$.

(iv) $\mathrm{g} \beta$ continuous if $f^{-1}(V)$ is $\mathrm{g} \beta$ open in $(X, \tau)$ for every open set $V$ in $(Y, \sigma)$.

Definition:- 2.4 [6] A subset $A$ of a Nano topological space $\left(U, \tau_{R}(X)\right)$ is called Nano generalized $\beta$ closed set (briefly $\mathrm{Ng} \beta$ closed) if $N \beta c l(A) \subseteq V$ whenever $A \subseteq V$ and $V$ is Nano open in $\left(U, \tau_{R}(X)\right)$.

Definition:- 2.5 [7] Let $\left(U, \tau_{R}(X)\right)$ and $\left(V, \sigma_{R^{\prime}}(Y)\right)$ be Nano topological spaces. Then a mapping $f:\left(U, \tau_{R}(X)\right) \rightarrow\left(V, \sigma_{R^{\prime}}(Y)\right)$ is called $\mathrm{Ng} \beta$ continuous on $U$ if the inverse image of every Nano open set in $V$ is $\mathrm{Ng} \beta$ open in $U$.

Definition:- 2.6 [2] A subset $A$ of a topological space $(X, \tau)$ is called generalized locally closed set (briefly GLC) if $A=G \cap F$ where $G$ is g open and $F$ is g closed in $X$.

3. Nano generalized $\beta$ locally closed sets:-

Definition:- 3.1 A subset $A$ of a Nano topological space $\left(U, \tau_{R}(X)\right)$ is called Nano generalized $\beta$ locally closed set (briefly NG $\beta$ LC) if $A=G \cap F$ where $G$ is $\mathrm{Ng} \beta$ open and $F$ is $\mathrm{Ng} \beta$ closed in $\left(U, \tau_{R}(X)\right)$.

Definition:- 3.2 A subset $A$ of a Nano topological space $\left(U, \tau_{R}(X)\right)$ is called (NG $\beta$ LC) ${ }^{*}$ set if $A=G \cap F$ where $G$ is $\operatorname{Ng} \beta$ open and $F$ is Nano closed in $\left(U, \tau_{R}(X)\right)$.

Definition:- 3.3 A subset $A$ of a Nano topological space $\left(U, \tau_{R}(X)\right)$ is called (NG $\beta$ LC) ${ }^{* *}$ set if $A=G \cap F$ where $G$ is Nano open and $F$ is $\mathrm{Ng} \beta$ closed in $\left(U, \tau_{R}(X)\right)$.

Definition:- 3.4 A subset $A$ of a Nano topological space $\left(U, \tau_{R}(X)\right)$ is called strongly Nano generalized $\beta$ locally closed set (briefly strongly NG $\beta$ LC) if $A=G \cap F$ where $G$ is strongly $\mathrm{Ng} \beta$ open and $F$ is strongly $\mathrm{Ng} \beta$ closed in $\left(U, \tau_{R}(X)\right)$.

Theorem:- 3.5 Every strongly NG $\beta$ LC set is NG $\beta$ LC set.

Proof:- Every strongly $\mathrm{Ng} \beta$ closed set is $\mathrm{Ng} \beta$ closed set proof follows.

Remark: 3.6:- The converse of the above theorem need not be true which is seen in the below example. 
Example:- 3.7 Let $U=\{a, b, c, d, e\}$ with $U / R=\{\{a, b\},\{c, e\},\{d\}\}$ and $X=\{a, d\}$. Then the Nano topology is defined as $\tau_{R}(X)=\{U, \phi,\{d\},\{a, b, d\},\{a, b\}\}$. Here the set $\{a, b, c, d\}$ is NG $\beta$ LC set but not strongly NG $\beta$ LC set in $U$.

Remark: 3.8:- Let $\left(U, \tau_{R}(X)\right)$ and $\left(V, \sigma_{R^{\prime}}(Y)\right)$ be Nano topological spaces. Then

(i) Every NLC set is NG $\beta$ LC set.

(ii) Every NGLC set NG $\beta$ LC set.

(iii) Every (NGLC) ${ }^{*}$ set is NG $\beta$ LC set.

(iv) Every (NGLC) ${ }^{* *}$ set is NG $\beta$ LC set.

(v) Every NLC set is (NG $\beta$ LC) ${ }^{*}$ set.

(vi) Every NLC set is (NG $\beta$ LC) ${ }^{* * *}$ set.

Similarly we prove for strongly NG $\beta$ LC set.

Theorem:- 3.9 If a subset $A$ of a Nano topological space $\left(U, \tau_{R}(X)\right)$ is (NG $\beta$ LC) ${ }^{*}$ set then there exist a $N g \beta$ open set $G$ such that $A=G \cap \beta c l(A)$.

Proof:- Assume that $A$ is (NG $\beta$ LC) ${ }^{*}$ set. Then there exist a $N g \beta$ open set $G$ and Nano closed set $F$ such that $A=G \cap F$. Since $A \subseteq F$ and $F$ is Nano closed, $A \subseteq N \beta c l(A) \subseteq F$. Then $A \subseteq G$ and $A \subseteq N \beta c l(A)$ andhence $A \subseteq G \cap \beta c l(A)$. To prove the reverse inclusion, if $x \in G \cap \beta c l(A)$ then $\quad x \in G$ and $x \in \beta c l(A) \subseteq F$. So $x \in G \cap F=A$. Hence $G \cap \beta c l(A) \subseteq A$. Therefore $A=G \cap \beta c l(A)$.

Theorem:- 3.10 For a subset of the following conditions.

i) $A \in N G \beta L C^{*}(U, X)$.

ii) $A=G \cap N \beta c l(A)$ for some $N g \beta$ open set G.

iii) $N \beta c l(A)-A$ is $N g \beta$ closed.

iv) $A \cup(U-N \beta c l(A))$ is $N g \beta$ closed.

are related as follows: i) and ii) are equivalent, iii) and iv) are equivalent and iv) implies ii).

\section{Proof:-}

i) $\Rightarrow$ ii) Let $A \in N G \beta L C^{*}(U, X)$. Then there exist a $\operatorname{Ng} \beta$ open set $G$ and a Nano closed set $F$ such that $A=G \cap F$. Since $A \subseteq G$ and $A \subseteq N \beta c l(A)$ we have $A=G \cap N \beta c l(A)$. Conversely since $N \beta c l(A) \subseteq F, G \cap N \beta c l(A) \subseteq G \cap F=A$ which implies that $A=G \cap N \beta c l(A)$.

ii) $\Rightarrow$ i) Since $G$ is $\operatorname{Ng} \beta$ open and $N \beta c l(A)$ is Nano closed $G \cap N \beta c l(A) \in N G \beta L C^{*}(U, X)$.

iii) $\Rightarrow$ iv) $\quad$ Let $\quad P=N \beta c l(A)-A \quad$. Then $\quad A \cup(U-N \beta c l(A)) \quad$ is $\quad \operatorname{Ng} \beta \quad$ open. Since $U-P=A \cup(U-N \beta c l(A))$ holds and hence $U-F$ is $\operatorname{Ng} \beta$ open.

iv) $\Rightarrow$ iii) Let $Q=A \cup(U-N \beta c l(A))$. Then $U-Q$ is $N g \beta$ closed and $U-Q=N \beta c l(A)-A$ holds.

iv) $\Rightarrow$ ii) Let $\quad G=A \cup(U-N \beta c l(A))$. Then $\quad$ is $\quad \operatorname{Ng} \beta \quad$ open. Now $G \cap N \beta c l(A)=[A \cup(U-N \beta c l(A))] \cap N \beta c l(A)=N \beta c l(A) \cap A \cup[N \beta c l(A) \cap(U-N \beta c l(A))]$ $=A \cup \phi=A$. Therefore $A=G \cap N \beta c l(A)$. 
Definition:- 3.11 A Nano topological space $\left(U, \tau_{R}(X)\right)$ is said to be a $\operatorname{Ng} \beta$ submaximal if every Nano dense subset of $\left(U, \tau_{R}(X)\right)$ is $\mathrm{Ng} \beta$ open in $\left(U, \tau_{R}(X)\right)$.

Theorem:- 3.12 Every Nano submaximal space is $\mathrm{Ng} \beta$ submaximal but bot conversely.

Proof: Let $U$ be a Nano submaximal and $A$ be a Nano dense subset of $U$. Then $A$ is Nano open. But every Nano open set is $\mathrm{Ng} \beta$ open and so $A$ is $\operatorname{Ng} \beta$ open. Therefore $U$ is $\mathrm{Ng} \beta$ submaximal.

Proposition:- 3.13 The following results are true for any two subsets $A$ and $B$ of a Nano topological space $\left(U, \tau_{R}(X)\right)$.

i) If $A, B \in N G \beta L C^{*}(U, X)$ then $A \cap B \in N G \beta L C^{*}(U, X)$.

ii) If $A \in N G \beta L C(U, X)$ and $B$ is $\operatorname{Ng} \beta$ open then $A \cap B \in N G \beta L C(U, X)$.

iii) If $A \in N G \beta L C^{*}(U, X)$ and $B$ is $\operatorname{Ng} \beta$ open then $A \cap B \in N G \beta L C^{*}(U, X)$.

iv) If $A \in N G \beta L C^{* *}(U, X)$ and $B$ is strongly $\operatorname{Ng} \beta$ open then $A \cap B \in N G \beta L C(U, X)$.

v) If $A \in N G \beta L C^{* *}(U, X)$ and $B$ is Nano open then $A \cap B \in N G \beta L C * *(U, X)$.

Theorem:- 3.14 A Nano topological space $\left(U, \tau_{R}(X)\right)$ is $\mathrm{Ng} \beta$ submaximal iff $N G \beta L C^{*}(U, X)=P(X)$.

Proof:- (Necessary) Let $A \in P(X)$. Let $G=A \cup(U-N \beta c l(A))$. Then $N \beta c l(G)=U$. Since $U$ is $\operatorname{Ng} \beta$ submaximal, $G$ is $\operatorname{Ng} \beta$ open. By theorem 3.11, $A \in N G \beta L C^{*}(U, X)$ and so $N G \beta L C^{*}(U, X)=P(X)$.

(Sufficient) Let $A$ be a Nano dense subset of $U$. Then $A \cup(U-N \beta c l(A))=A \cup \phi=A$. Since $A \in N G \beta L C^{*}(U, X)$, by theorem $3.11 A$ is $\operatorname{Ng} \beta$ open in $U$. Hence $U$ is $\mathrm{Ng} \beta$ submaximal.

\section{4. $\mathrm{Ng} \beta$ lc continuous map and $\mathrm{ng} \beta$ lc irresolute map:-}

Definition:- 4.1 A function $f:\left(U, \tau_{R}(X)\right) \rightarrow\left(V, \sigma_{R^{\prime}}(Y)\right)$ is called NG $\beta$ LC continuous (NG $\beta$ LC* continuous and $\mathrm{NG} \beta \mathrm{LC}^{* *}$ continuous)if $f^{-1}(G) \in N G \beta L C(U, X)\left(f^{-1}(G) \in N G \beta L C *(U, X)\right.$ and $f^{-1}(G) \in N G \beta L C * *(U, X)$ ) for every Nano open set $G$ of $V$.

Theorem:- 4.2 Afunction $f: U \rightarrow V$ be a function then we have the following.

i) If $f$ is NLC continuous then $f$ is $\mathrm{NG} \beta$ LC continuous (NG $\beta$ LC* continuous and $\mathrm{NG} \beta$ LC** continuous).

ii) If $f$ is NG $\beta$ LC* continuous or NG $\beta$ LC** continuous then $f$ is $\mathrm{NG} \beta$ LC continuous.

Proof: i) Suppose that $f$ is NLC continuous. Let $G$ be a Nano open set in $U$. Then $f^{-1}(G)$ is NLC set in $U$. Since every NLC set is NG $\beta$ LC set (NG $\beta$ LC* set and NG $\beta$ LC** set ), it follows that $f$ is NG $\beta$ LC continuous (NG $\beta$ LC* continuous and NG $\beta$ LC** continuous).

ii)Let $f: U \rightarrow V$ be NG $\beta$ LC* continuous or NG $\beta$ LC** continuous. Since every NG $\beta$ LC* set or NG $\beta$ LC** set is NG $\beta$ LC set. Therefore $f$ is NG $\beta$ LC continuous.

Theorem:- 4.3 If $f: U \rightarrow V$ is NG $\beta$ LC continuous and $g: V \rightarrow W$ is Nano continuous, then $g \circ f: U \rightarrow W$ is NG $\beta$ LC continuous. 
Proof:- Let $F$ be Nano closed in $W$. Then $g^{-1}(F)$ is Nano closedin $V$, since $g$ is Nano continuous and then $f^{-1}\left(g^{-1}(F)\right)=(g \circ f)^{-1}(F)$ is NG $\beta$ LC set in $U$, as $f$ is NG $\beta$ LC continuous. Thus $g \circ f$ is NG $\beta$ LC continuous.

Theorem:- 4.4 If $f: U \rightarrow V$ is $\mathrm{NG} \beta$ LC* continuous and $g: V \rightarrow W$ is Nano continuous, then $g \circ f: U \rightarrow W$ is $\mathrm{NG} \beta \mathrm{LC} *$ continuous.

Theorem:- 4.5 If $f: U \rightarrow V$ is $\mathrm{NG} \beta \mathrm{LC}^{* *}$ continuous nd $g: V \rightarrow W$ is Nano continuous, then $g \circ f: U \rightarrow W$ is NG $\beta$ LC** continuous.

Proof of the theorem 4.4 and 4.5 are similar to the theorem 4.3.

Definition:- 4.6 A function $f:\left(U, \tau_{R}(X)\right) \rightarrow\left(V, \sigma_{R^{\prime}}(Y)\right)$ is called NG $\beta$ LC irresolute (NG $\beta$ LC* irresolute and $\quad \mathrm{NG} \beta \mathrm{LC}^{* *} \quad$ irresolute $) \quad$ if $\quad f^{-1}(G) \in N G \beta L C(U, X)\left(f^{-1}(G) \in N G \beta L C^{*}(U, X)\right.$ and $\left.f^{-1}(G) \in N G \beta L C^{* *}(U, X)\right) \quad$ for $\quad$ every $\quad G \in N G \beta L C(V, Y)(G \in N G \beta L C *(V, Y)$ and $G \in N G \beta L C *(V, Y)$ ).

Theorem:- 4.7 A function $f: U \rightarrow V$ be a function then we have the following.

i) If $f$ is NLC irresolute then $f$ is NG $\beta$ LC irresolute (NG $\beta$ LC* irresolute and NG $\beta$ LC** irresolute).

ii) If $f$ is NG $\beta$ LC irresolute (NG $\beta$ LC* irresolute or NG $\beta$ LC** irresolute) then $f$ is NG $\beta$ LC continuous (NG $\beta$ LC* continuous and NG $\beta$ LC** continuous).

Proof: i) Suppose that $f$ is NLC continuous. Let $G$ be a NLC set in $U$. Then $f^{-1}(G)$ is NLC set in $U$. Since every NLC set is NG $\beta$ LC set (NG $\beta$ LC* set and NG $\beta$ LC** set), it follows that $f$ is NG $\beta$ LC irresolute (NG $\beta \mathrm{LC} *$ irresolute and $\mathrm{NG} \beta \mathrm{LC} * *$ irresolute) .

ii)Let $G$ be a Nano open set in $U$ and $G$ is NG $\beta$ LC set in $V$. Then $f^{-1}(G) \in N G \beta L C(U, X)$ as $f$ is NG $\beta$ LC irresolute (NG $\beta$ LC* irresolute or NG $\beta$ LC** irresolute). Hence $f$ is NG $\beta$ LC continuous (NG $\beta$ LC* continuous and $\mathrm{NG} \beta \mathrm{LC} * *$ continuous).

Theorem:- 4.8 If $f: U \rightarrow V$ is $\mathrm{NG} \beta$ LC irresolute and $g: V \rightarrow W$ is NG $\beta$ LC continuous, then $g \circ f: U \rightarrow W$ is NG $\beta$ LC continuous.

Proof: Let $F$ be Nanoclosedin $W$. Then $g^{-1}(F)$ is NG $\beta$ LC setin $V$, since $g$ is NG $\beta$ LC continuous. Again since $f$ is NG $\beta$ LC irresolute, $f^{-1}\left(g^{-1}(F)\right)=(g \circ f)^{-1}(F)$ is NG $\beta$ LC set in $U$. Thus $g \circ f$ is $\operatorname{Ng} \beta$ LC continuous.

Theorem:- 4.9 If $f: U \rightarrow V$ is $\mathrm{NG} \beta$ LC* irresolute and $g: V \rightarrow W$ is $\mathrm{NG} \beta \mathrm{LC}^{*}$ continuous, then $g \circ f: U \rightarrow W$ is NG $\beta$ LC* continuous.

Theorem: 4.10 If $f: U \rightarrow V$ is $\mathrm{NG} \beta \mathrm{LC}^{* *}$ irresolute and $g: V \rightarrow W$ is $\mathrm{NG} \beta \mathrm{LC}^{* *}$ continuous, then $g \circ f: U \rightarrow W$ is $\mathrm{NG} \beta \mathrm{LC}^{* *}$ continuous.

Proof of the theorem 4.9 and 4.10 are similar to the theorem 4.8.

Similarly we can prove the following theorems. 
Theorem:- 4.11 If $f: U \rightarrow V$ is $\mathrm{NG} \beta$ LC irresolute and $g: V \rightarrow W$ is NG $\beta$ LC irresolute, then $g \circ f: U \rightarrow W$ is NG $\beta$ LC irresolute.

Theorem:- 4.12 If $f: U \rightarrow V$ is $\mathrm{NG} \beta$ LC* irresolute and $g: V \rightarrow W$ is $\mathrm{NG} \beta$ LC* irresolute, then $g \circ f: U \rightarrow W$ is NG $\beta$ LC* irresolute.

Theorem:- 4.13 If $f: U \rightarrow V$ is $\mathrm{NG} \beta \mathrm{LC}^{* *}$ irresolute and $g: V \rightarrow W$ is $\mathrm{NG} \beta$ LC L* $^{*}$ irresolute, then $g \circ f: U \rightarrow W$ is NG $\beta$ LC** irresolute.

Theorem:- 4.14 If $f: U \rightarrow V$ is NG $\beta$ LC irresolute and $g: V \rightarrow W$ is NLC continuous, then $g \circ f: U \rightarrow W$ is NG $\beta$ LC continuous.

Theorem:- 4.15 If $f: U \rightarrow V$ is NG $\beta$ LC irresolute and $g: V \rightarrow W$ is $\operatorname{Ng} \beta$ continuous, then $g \circ f: U \rightarrow W$ is $\mathrm{NG} \beta \mathrm{LC}$ continuous

Theorem:- 4.16 If $f: U \rightarrow V$ is $\mathrm{NG} \beta$ LC* irresolute and $g: V \rightarrow W$ is $\mathrm{NG} \beta$ LC* continuous, then $g \circ f: U \rightarrow W$ is NG $\beta$ LC* continuous.

Theorem:- 4.17 If $f: U \rightarrow V$ is $\mathrm{NG} \beta$ LC** irresolute and $g: V \rightarrow W$ is $\mathrm{NG} \beta$ LC** continuous, then $g \circ f: U \rightarrow W$ is NG $\beta$ LC** continuous.

\section{References:-}

1. Abd EL-Monsef M. E., EL-Deep S. N. and Mahmoud R. A., $\beta$-Open Sets and $\beta$ continuous mappings, Bull. Fac. Sci., Assiut Univ., 12 (1983), 77-90.

2. Balachandran, K., Sundaram, P. and Maki, H., Generalized locally closed sets and GLC-continuous functions, Indian J. Pure. Appl. Math., 27(1996), 235-244.

3. Gnanmbal. Y., On Nano $\beta$ open sets, Int. Jr. of Engineering, 1(2) (2015), 1-6.

4. LellisThivagar .M., and Carmel Richard, On Nano forms of weakly open sets, International

5. Journal of Mathematics and Statistics Invention, 1(1) (2013), 31 -37.

6. LellisThivagar .M., and Carmel Richard, On Nano Continuity, Mathematical Theory and Modeling, 3(7)(2013), 32-37.

7. Shalini. S.B., and Indirani. K., Characterisation of nano generalized $\beta$ closed sets in nano topological spaces, International Journal Sciences and Applied Research, 4(1)(2017), 7-11.

8. Shalini. S.B., and Indirani. K., On Nano generalized $\beta$ continuous functions and Nano generalized $\beta$ irresolute functions in Nano topological spaces, IOSR Journal of Mathematics (IOSR-JM), 13(1)(2017), 79-86. 